\title{
Biomarkers of benefit from cetuximab-based therapy in metastatic colorectal cancer: interaction of EGFR ligand expression with RAS/RAF, PIK3CA genotypes
}

George Pentheroudakis ${ }^{1 *}$, Vassiliki Kotoula ${ }^{2,3}$, Wendy De Roock ${ }^{4}$, George Kouvatseas $^{5}$, Pavlos Papakostas ${ }^{6}$, Thomas Makatsoris ${ }^{7}$, Demetris Papamichael ${ }^{8}$, loannis Xanthakis ${ }^{9}$, Joseph Sgouros ${ }^{10}$, Despina Televantou ${ }^{3}$, Georgia Kafiri ${ }^{11}$, Athanassios C Tsamandas ${ }^{12}$, Evangelia Razis ${ }^{13}$, Eleni Galani ${ }^{14}$, Dimitrios Bafaloukos ${ }^{15}$, Ioannis Efstratiou ${ }^{16}$, Iliada Bompolaki ${ }^{17}$, Dimitrios Pectasides ${ }^{18}$, Nicholas Pavlidis ${ }^{1}$, Sabine Tejpar $^{4}$ and George Fountzilas ${ }^{9}$

\begin{abstract}
Background: More than half of patients with KRAS-wild type advanced colorectal cancer (CRC) fail anti-EGFR monoclonal antibodies. We studied EGFR-axis messenger RNA (mRNA) expression and RAS, RAF, PIK3CA mutations in order to identify additional biomarkers of cetuximab efficacy.

Methods: Previously genotyped (KRAS, NRAS, BRAF, PIK3CA mutations) formalin-fixed paraffin-embedded tumour biopsies of 226 cetuximab-treated CRC patients (1st to 3rd line therapy) were assessed for mRNA expression of epidermal growth factor receptor (EGFR) and its ligands EGF, Transofrming Growth Factor-a (TGFA), Amphiregulin (AREG) and Epiregulin (EREG) with real time quantitative PCR. Mutations were detected in 72 (31.9\%) tumours for KRAS, in 6 (2.65\%) for BRAF, in 7 (3.1\%) for NRAS and in 37 (16.4\%) for PIK3CA.
\end{abstract}

Results: Only PIK3CA mutations occasionally coexisted with other gene mutations. In univariate analysis, prognostic significance for survival ( from metastases until death) was seen for BRAF mutations (Hazard Ratio HR 8.1, 95\% Cl 3.4-19), codon 12-only KRAS mutations (HR 1.62, 95\% Cl 1.1-2.4), high AREG mRNA expression only in KRAS wild type CRC (HR 0.47, 95\% Cl 0.3-0.7) and high EREG mRNA expression irrespective of KRAS mutation status (HR 0.45, $95 \% \mathrm{Cl}$ 0.28-0.7). EREG tumoural mRNA expression was significantly associated with a 2.26-fold increased likelihood of objective response to cetuximab therapy (RECIST 1.1). In multivariate analysis, favourable predictive factors were high AREG mRNA in KRAS wild type tumours, high EREG mRNA, low Ephrin A2 receptor mRNA. Cetuximab-treated patients with AREG-low KRAS wild type CRC fared very poorly, their survival being similar to KRAS mutant CRC. Patients with KRAS codon 13 or other non-codon 12 mutations had a median survival (30 months, 95\% Cl 20-35) similar to that of patients with KRAS wild-type (median survival 29 months, 95\% Cl 25-35), in contrast to patients with KRAS codon 12 mutations who fared worse (median survival 19 months, 95\% Cl 15-26).

Conclusions: BRAF and codon 12 KRAS mutations predict for adverse outcome of CRC patients receiving cetuximab. AREG mRNA reflects EGFR signalling in KRAS wild type tumours, predicting for cetuximab efficacy when high and failure when low. EREG may have a prognostic role independent of KRAS mutation.

Keywords: Cetuximab, Epidermal growth factor receptor, EGFR ligands, KRAS, BRAF, PI3K gene mutations, Biomarkers

\footnotetext{
* Correspondence: gpenther@otenet.gr

${ }^{1}$ Department of Medical Oncology, loannina University Hospital, Ioannina,

Greece

Full list of author information is available at the end of the article
} 


\section{Background}

Colorectal cancer (CRC) is among the «big killers» in populations of developed societies, with a reported death toll of 50,000 yearly in the United States [1]. Recent advances in modern therapeutic strategies resulted in significant survival improvement of patients with metastatic disease. The Epidermal Growth Factor Receptor (EGFR) on the cancer cell surface relays signals of proliferation, angiogenesis, metastasis and antibodies binding it have been partly responsible for the observed outcomes improvement [2]. Cetuximab, a chimeric IgG1 monoclonal antibody (moAb) and panitumumab, a humanised IgG2 moAb are currently licensed for the treatment of patients with metastatic colorectal cancer either in combination with chemotherapy in the first and second line setting or as monotherapy for refractory disease. The need to identify tumours addicted to EGFR signalling and thus amenable to anti-EGFR therapeutic modulation became apparent early on, as response rates to cetuximab regimens in unselected patient populations were typically lower than 30\% [3].

KRAS is a cytoplasmic GTP-binding protein with low inherent GTPase activity. When the KRAS protein is bound to GTP, it relays signals of cellular proliferation and inhibition of apoptosis, acting as a typical oncogene. KRAS mutations were observed mainly in gene exon 2, resulting in abrogated GTPase activity and locking the KRAS protein in the active KRAS-GTP conformation. By activating the RAS/RAF/MAPK axis downstream of EGFR, these mutations render therapeutic modulation of EGFR irrelevant [4]. Indeed, clinical data confirmed the predictive value of KRAS exon 2 mutations for resistance to cetuximab and panitumumab, leading to the license of these moAbs exclusively for the management of patients with KRAS-wild type colorectal cancers [5-7]. Despite application of such a «negative selection» biomarker, the KRAS-wild type patient population benefits from anti-EGFR strategies in less than half of cases. Research efforts towards identification of additional predictive biomarkers have generated interesting, though preliminary and at times conflicting data on the importance of tumour mRNA levels of EGFR ligands, of activating mutations in other genes such as BRAF, PIK3CA [8-11]. Finally, the codon localisation of KRAS mutations was found to possess differential transforming potential in cell cultures and to bear distinct predictive value for cetuximab resistance in clinical series [9].

We report a retrospective translational research project on previously diagnosed formalin-fixed paraffin-embedded (FFPE) colorectal carcinomas from 226 patients who were treated with cetuximab-based therapy in the first, second or third line setting for metastatic disease. In the context of a broad translational research protocol involving exploratory analyses of multiple biomarkes, our EGFR axis project aimed at screening for biomarkers of cetuximab benefit. It included studying gene expression of EGFR, its ligands epidermal growth factor (EGF), amphiregulin (AREG), epiregulin (EREG), transforming growth factor-a (TGFa) and the presence of activating mutations in KRAS, BRAF, NRAS, PIK3CA genes as well as the possible distinct effect of KRAS and PIK3CA mutations at various codons.

\section{Methods}

\section{Patient selection}

Two hundred and twenty-six patients with histologically established diagnosis of colorectal adenocarcinoma, for whom collection of tissue samples was part of their routine care and treatment, provided informed consent for the research use of their biologic material. The retrospective translational research protocol was approved by the Ethics Committee/Scientific Council of the General Hospital Papageorgiou, Thessaloniki (Meeting number 158/26-6-12). The study was performed in compliance with NCI-EORTC's REMARK recommendations. Upon development of metastatic disease, these patients were managed with cetuximab-based therapy from May 2004 until December 2008 in Hellenic Cooperative Oncology Group (HeCOG)- affiliated centers. All FFPE blocks were retrospectively identified and quality-controlled by an experienced pathologist (Despina Televantou, DT) for the histological diagnosis of colorectal adenocarcinoma and for the assessment of tumour cell content. In case of less than $50 \%$ tumour cells in whole sections, manual tumour macrodissection was applied before further processing. Thus, all molecular samples contained molecular templates (DNA, RNA) above the 50\% threshold.

\section{Tissue material and molecular studies}

In total, 226 tissue blocks were available for DNA analysis. DNA samples had been centrally assessed for KRAS, NRAS, BRAF and PIK3CA mutations by using a Sequenom MALDI-TOF MassARRAY multiplex PCR method and the Sequenom MassARRAY Assay Design 3.1 software as previously published [8].

Corresponding sections or macrodissected tissue fragments from 199 available tissue blocks were lysed overnight and processed for RNA extraction with TRIZOL-LS (Invitrogen/Life Technologies, Paisley, UK), according to the manufacturers' instructions. RNA was reverse transcribed with Superscript III and random hexamers (Invitrogen/Life Technologies). cDNAs were normalized at $25 \mathrm{ng} / \mathrm{ul}$ and stored at $-20^{\circ} \mathrm{C}$ until use. mRNA expression was assessed with Taqman-MGB assays (Applied Biosystems/Life Technologies) for exon spanning amplicons, for the following targets with data in parentheses corresponding to assay ID, reference sequence, location and size of amplicon, in the same order: AREG 
(Hs00950669_m1, NM_001657.2, exons 3-4, 66 bp); EGF (Hs01099999_m1, NM_001963.3, exons 20-21, 70 bp); EGFR (Hs00193306_m1, NM_005228.3, exons 20-21, 69 bp); EREG (Hs00914313_m1, NM_001432.2, exons 34, 65 bp); and, TGFa (Hs00608187_m1, NM_001099691.1, NM_003236.2, exons 4-5, 70 bp).

Samples were run in duplicates in an ABI7900HT real time PCR system along with negative (no-template) and positive controls (commercially available reference RNA: TaqMan ${ }^{\circledR}$ Control Total RNA, cat. no 4307281, Applied Biosystems/Life Technologies). As an endogenous control and for the normalization of CT (cycle threshold) values, an assay targeting GUSB mRNA (beta-glucuronidase [\#4333767 F]) was used. GUSB was preferred over usually applied endogenous controls because (a) no pseudogenes have as yet been reported for this gene, and (b) it has been identified as one among the best preserved mRNA targets in FFPE tissues [12]. To obtain linear Relative Quantification (RQ) values, relative expression was assessed as (40$\mathrm{dCT}$ ), as previously described, whereby $\mathrm{dCT}$ (or deltaCT) was calculated as (average target CT) - (average GUSB CT) from all eligible measurements [13]. Samples were considered eligible for analysis when both GUSB CTs in duplicates were $<36$ and when duplicate dRQ values (dRQ1 - dRQ2) were $<0.85$. As observed upon consecutive runs of 30 eligible samples (15\% of all samples), minimummaximum deltaRQs for all assays were <1.5 (AREG: 0.43; EGF: 0.85; EGFR: 1.2; EREG: 0.69; TGFa: 0.64). RNA studies were performed in the Laboratory of Molecular Oncology, Aristotle University of Thessaloniki, Greece.

\section{Endpoints}

The study endpoints were a) Survival, from the time of diagnosis of metastatic disease until death or last follow-up, b) Best objective response rate (ORR) to any line of cetuximabbased therapy by RECIST 1.1 criteria [14]. A progressionfree survival endpoint was not used intentionally because in the setting of cetuximab use in various lines of therapy, it could be influenced by the distribution of cetuximab therapy line in various categories of biomarkers under study.

We analysed the prognostic/predictive impact of different types of mutations of KRAS (codon 12 versus codon 13 versus other mutations versus wild type) and PIK3CA genes (exon 9 versus exon 20 versus wild-type). We also compared outcomes of cases with complex mutational phenotypes for prognostic/predictive utility. Complex Genotype comparison 1 (CG1) compared outcomes of KRAS wild type versus KRAS mutant versus [PIK3CA mutant + (KRAS or BRAF mutant)]. CG comparison 2 (CG2) compared outcomes of PIK3CA wild type versus PIK3CA mutant versus [PIK3CA mutant + (KRAS or BRAF mutant)], while CG comparison 3 (CG3) outcomes of all KRAS, BRAF, PIK3CA wild type versus any of them mutant versus [PIK3CA mutant + (KRAS or BRAF mutant)].

\section{Statistical analysis}

Cut-offs for each mRNA marker were selected either as natural breaks in the distributional profiles of the corresponding RQ values or, if these were not apparent, as RQ value quartiles, which were examined for statistically significant associations with survival at the 5\% significance level. If a cut-off was statistically significant it was used to classify low and high expressing tumours. In the absence of a candidate cut-off, mRNA markers were examined as continuous variables. The criterion for selecting cut-offs was the minimum p-value method. In cases of more than one plausible candidate points, graphical diagnostic plots using martingale residuals were used for time to event endpoints and the method of Miller and Seigmund for ORR [15]. Time-to -event distributions were estimated using Kaplan-Meier curves. Associations between biomarkers, mutations and basic patient and tumour characteristics were examined using the Fisher's exact test or the Chi-Square test. Cox proportional hazards and logistic regression were used for univariate survival and ORR analyses respectively. Significant correlations between parameters were tested as interactions for prognostic significance in terms of survival and ORR. The multivariate model contained every significant variable and interaction from univariate analyses. A backward selection procedure with $15 \%$ removal cut-off was used.

The major aim of our research proposal is the characterization of molecular biomarkers which would help to predict survival to cetuximab therapy. The study's power justification scheme was based upon the following assumptions: a) expected follow up of 60 months, based on 56 months accrual duration with 2 years additional follow-up period b) ratio of expressed to non-expressed samples of either 1:3, 1:1 or $3: 1$ and c) HR of 0.4-0.5. With this background information and risk assumptions the sample of 226 patients provided power from $91 \%$ to $99 \%$ at the $5 \%$ level of significance for performing a two sided log-rank test.

\section{Results}

\section{Patient and tumour demographics}

A total of 226 patients of a median age of 62.6 years (range 26-85) underwent excisional or incisional biopsy of colorectal adenocarcinoma, of whom 83 (36.8\%) were diagnosed with localised (stage I-III) colon cancer and 137 (60.6\%) with metastatic disease (Table 1). The primary tumour was located in the left colon (distal transverse to rectum) in 165 (73.0\%) of cases and in the right colon (caecum to proximal transverse) in 60 (26.6\%). Rectal tumours accounted for 71 cases (31\%).

From May 2004 until December 2008, cetuximab had been administered as an intravenous infusion according to standard regimens (loading dose $400 \mathrm{mg} / \mathrm{m} 2$ followed by weekly $250 \mathrm{mg} / \mathrm{m} 2)$ as monotherapy $(42,13.4 \%)$ or with regimes based on irinotecan $(153,48.7 \%)$, oxaliplatin (84, $26.7 \%)$ or both agents $(29,9.2 \%)$. The lines of therapy 
Table 1 Disease demographics and management data

\begin{tabular}{ll}
\hline Parameter & N (\%) \\
\hline Age (median) & 62.6 years \\
Gender (Female/Male) & $97(43.0 \%) / 129(57.0 \%)$ \\
Histological Grade & \\
$1-2$ & $170(75.2 \%)$ \\
$3-4$ & $46(20.4 \%)$ \\
Obstruction Yes/No & $30(13.2 \%) / 179(79.2 \%)$ \\
Perforation Yes/No & $9(4.0 \%) / 201(89.0 \%)$ \\
Primary Site Left/Right & $165(73.0 \%) / 60(26.6 \%)$ \\
Rectum & $71(31.0 \%)$
\end{tabular}

TNM stage at biopsy

I-III versus IV

Median Follow Up

Deaths

Line of therapy at Cetuximab

administration

1st line

2nd line

3rd line and beyond

Type of therapy at Cetuximab administration

Irinotecan-based

Oxaliplatin-based

Both irinotecan and oxaliplatin

Only fluoropyrimidine

Single-agent Cetuximab

Objective Response to Cetuximab

All lines of therapy

Complete Respone (CR)

Partial Response (PR)

ORR (CR+PR)

Stable disease (SD)

Progressive disease (PD)

ORR by line of Cetuximab therapy

1st line

2nd line

3rd line

during which cetuximab was administered were 1st line in 38 (16.8\%), 2nd line in 108 (47.8\%), 3rd and beyond in 80 (35.4\%) (Table 1). Of note, as the period of therapy extended from 2004 until December 2008, an unselected patient population received cetuximab-based therapies, irrespective of KRAS mutational status.

mRNA markers and somatic genotypes

Out of the 199 samples tested for mRNA expression, three were found not eligible for all targets. In total,
84.4\% of samples were eligible for AREG analysis; $83.9 \%$ for EGF; 84.4\% for EGFR; 80.4\% for EREG; and 81.4\% for TGFa. Out of the 226 DNA samples screened for the presence of mutations, genotyping was successful in 205 samples for KRAS (90.8\%), 159 (70.4\%) for NRAS, 220 for BRAF and 220 for PIK3CA (97.3\% in each case) (Table 2). KRAS mutations were detected in 72 tumours (31.9\%), mostly in exon 2, in codon 12 in 45 cases (20.0\%) and in codon 13 in 16 cases (7.0\%), while BRAF V600E mutations were found in 6 cases $(2.6 \%)$. NRAS mutations were also quite rare, found only in 7 cases

Table 2 mRNA biomarkers and gene mutation status

\begin{tabular}{|c|c|c|c|}
\hline $\begin{array}{l}\text { Biomarkers } \\
\text { and } \\
\text { mutations }\end{array}$ & N (\%) & $\begin{array}{l}\text { Median } \\
\text { RQ }\end{array}$ & $\begin{array}{l}\text { Interquartile } \\
\text { range (IQR) }\end{array}$ \\
\hline AREG & 168 & 39.9 & $38.7-40.9$ \\
\hline EREG & 160 & 36.4 & $34.7-37.9$ \\
\hline EGF & 167 & 29.3 & 27.9-33.6 \\
\hline TGFa & 162 & 37.1 & $36.2-37.9$ \\
\hline EGFR & 168 & 38.3 & $37.7-39.2$ \\
\hline \multicolumn{4}{|l|}{ KRAS N=205 } \\
\hline All mutations & 72 (31.9\%) & & \\
\hline G12 & 45 (20.0\%) & & \\
\hline G13 & 16 (7.0\%) & & \\
\hline Q61 & $4(1.7 \%)$ & & \\
\hline A146 & $7(3.1 \%)$ & & \\
\hline Wild type & $\begin{array}{l}133 \\
(58.8 \%)\end{array}$ & & \\
\hline Missing & $21(9.3 \%)$ & & \\
\hline \multicolumn{4}{|l|}{ NRAS N=159 } \\
\hline All mutations & 7 (3.1\%) & & \\
\hline Q61 & $5(2.2 \%)$ & & \\
\hline G12 & $2(0.9 \%)$ & & \\
\hline Wild type & $\begin{array}{l}152 \\
(67.3 \%)\end{array}$ & & \\
\hline Missing & 67 (29.6\%) & & \\
\hline \multicolumn{4}{|l|}{ BRAF $N=220$} \\
\hline V600E & $6(2.6 \%)$ & & \\
\hline Wild type & $\begin{array}{l}214 \\
(94.7 \%)\end{array}$ & & \\
\hline Missing & $6(2.6 \%)$ & & \\
\hline \multicolumn{4}{|c|}{ PIK3CA N=220 } \\
\hline All mutations & $37(16.4 \%)$ & & \\
\hline Exon 9 & $20(8.8 \%)$ & & \\
\hline Exon 20 & $5(2.2 \%)$ & & \\
\hline Other & 12 (5.3\%) & & \\
\hline Wild type & $\begin{array}{l}183 \\
(81.0 \%)\end{array}$ & & \\
\hline Missing & $6(2.6 \%)$ & & \\
\hline
\end{tabular}


(3.1\%). PIK3CA mutations were detected in 37 tumours (16.4\%), 20 in exon $9(8.8 \%)$ and only five in exon 20 $(2.2 \%)$. This genotypic analysis was included in a previous publication [8].

Correlation analysis revealed that mutations in KRAS, BRAF, NRAS genes were mutually exclusive in all evaluable cases. On the contrary, mutations in the PIK3CA gene co-existed with KRAS mutations in 17 cases and with NRAS mutations in two. Statistically significant associations at the 2-sided $\mathrm{p}<0.05$ level were seen between wild type status of KRAS, BRAF genes and high AREG, EREG, EGFR mRNA levels. Among KRASwild type cases, $81.4 \%$ were seen in AREG-high and $57.4 \%$ in EREG-high tumours, while of KRAS-mutated cases only $63.9 \%$ were observed in AREG-high and only 35.7\% in EREG-high tumours. Similarly, among BRAFwild type cases, $76.1 \%$ were seen in AREG-high and $50.7 \%$ in EREG-high tumours, while of BRAF-mutated cases only two out of six (33.3\%) were observed in AREG-high and none in EREG-high tumours. Regarding the primary location, $64.8 \%$ of KRAS mutations $(\mathrm{p}=0.07)$ and $58 \%$ of PIK3CA mutations $(\mathrm{p}=0.036)$ occurred in left-sided colorectal tumours, whereas four (66.7\%) of BRAF mutations in right-sided colon carcinomas $(\mathrm{p}=0.024)$. mRNA RQ values were examined as continuous variables and significant correlations at Spearmann's $\mathrm{R}>0.4$ were found between AREG and EREG mRNA ( $\mathrm{R}=0.63$, 95\% CI 0.53-0.72, $\mathrm{p}=0.0005$ ).

\section{Factors with predictive significance at univariate analysis}

At a median follow-up of 73.6 months, 215 patients (95.1\%) had experienced disease progression and 163 (93.1\%) had died. The median survival was was 27 months (95\% CI 25-31). At univariate analysis, in the present cohort of patients treated with cetuximab the presence of BRAF mutations was significantly associated with an 8.1-fold increased risk of death compared to patients harbouring BRAF-wild type tumours. When all types of mutations were pooled for each gene, KRAS (HR 1.28, p=0.14), NRAS (HR 1.43, p=0.36) and PIK3CA (HR 1.27, $\mathrm{p}=0.25$ ) mutations did not have prognostic/ predictive utility. However, when types of mutations were analysed separately for each gene, KRAS codon 12 mutations were predictive for increased risk of death (HR 1.62, $\mathrm{p}=0.014$ ).

Among parameters studied for gene expression, only the 25th percentile of AREG mRNA values and the 75th percentile of EREG mRNA values dichotomized cases into groups with different outcome and were selected as optimal cut-offs. None of EGF, TGFa, EGFR mRNA levels disclosed cut-off points of prognostic significance. Cetuximab therapy in patients harbouring AREG-high (HR 0.47, p=0.0002) or EREG-high (HR 0.45, p=0.0009) tumours resulted in reduced risk of death. EGFR, EGF,
TGFa mRNA expression had no significant prognostic/ predictive value, either in the entire cohort or in the KRAS-wild type versus KRAS mutant cases.

Regarding objective tumour response to any line of cetuximab-based therapy, tumoural AREG and EREG mRNA expression levels were associated with tumour shrinkage as continuous variables. AREG was not statistically significant as categorical variable but EREG mRNA at the 75th percentile was associated with a 2.26fold increased likelihood of tumour response to cetuximab compared to tumours with lower EREG mRNA expression. AREG failed to predict response in either KRAS wild type or mutated cases. The expression levels of EREG in the tumours of responding and non-responding patients by KRAS gene status is depicted in a waterfall plot (Figure 1). Of note, in an exploratory analysis, the predictive significance for objective response was maintained only in KRAS mutated CRC, in which EREG had an odds ratio for response of 5.4 (95\% CI 1.2-23.8, $\mathrm{p}=0.024)$. By contrast, in KRAS-wild type tumours EREG failed to significantly predict for response to therapy (OR 2.2, 95\% CI 0.83-5.86, $\mathrm{p}=0.11$ ). EGF, TGFa and EGFR mRNA expression had no predictive utility for response to cetuximab in the whole cohort, neither in KRAS wild-type or KRAS mutated cases. All factors with potential predictive utility for cetuximab benefit are summarized in Table 3 .

\section{Interactions and factors with predictive utility at multivariate analysis}

Significant interactions (at the $1 \%$ significance level) between study variables for prognostic/predictive utility (survival, ORR) were sought. In terms of impact on survival, significant interactions were found between AREG mRNA levels and mutational status of the KRAS gene $(\mathrm{p}=0.0033)$. High mRNA levels of AREG predicted for longer survival in patients with KRAS wild-type (median survival 33 vs. 15 months, $\mathrm{p}=0.0005$, Figure 2), but not in KRAS mutant tumours (median survival 22 vs. 17 months, $\mathrm{p}=0.64$ ). In contrast, high mRNA levels of EREG were associated with favourable prognosis irrespective of KRAS mutational status. In KRAS wild type cases, the median survival was 37 months for EREG-high as compared to 23 months for EREG-low expressing tumours $(p=0.01)$, while in KRAS mutated tumours median survival was 33 versus 19 months $(\mathrm{p}=0.02)$ in EREGhigh vs. low cases (Figure 3). Similar findings for EREG high vs. low were seen when we examined complex cancer genotypes: a) KRAS+BRAF, both wild type vs. any mutant, b) KRAS+BRAF+PIK3CA+NRAS, all wild type vs. any mutant. AREG mRNA had a favourable predictive significance in wild type cases only, whereas EREG mRNA preserved its significance in wild type as well as in mutant cases.

A multivariate analysis was performed in the context of a broader mRNA profiling project of several biomarkers 


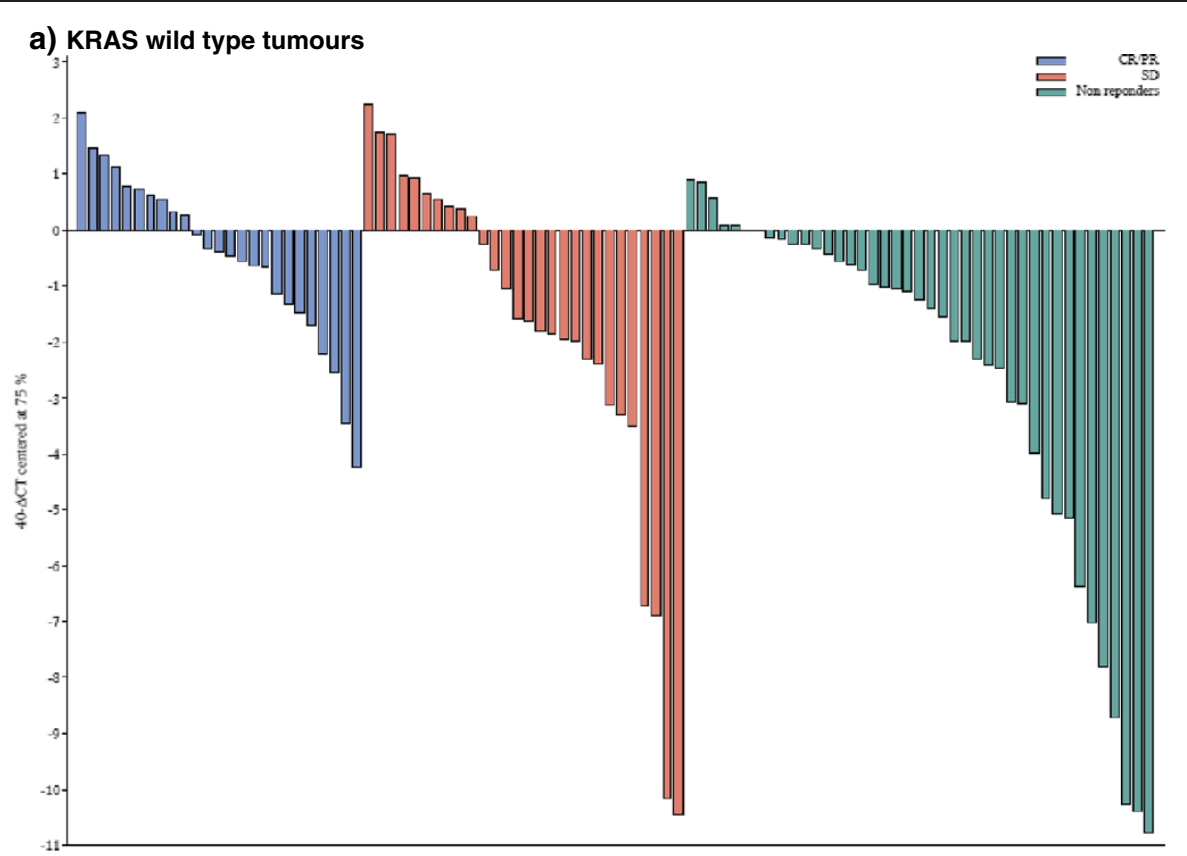

\section{b) KRAS mutant tumours}

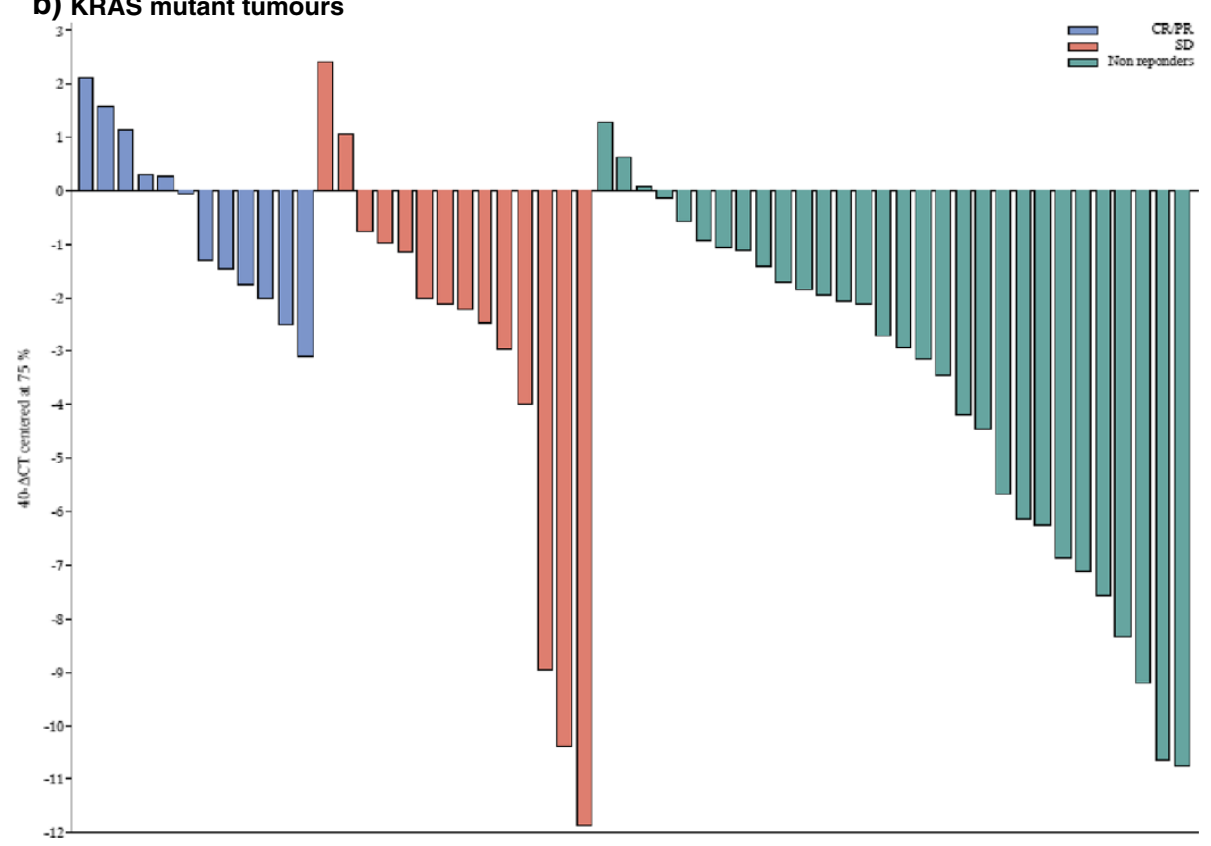

Figure 1 Waterfall plot of tumoural EREG mRNA levels in responding and non-responding patients by KRAS mutation status. a) KRAS wild type tumours, b) KRAS mutant tumours. X-axis corresponds to the 75th percentile EREG mRNA RQ values.

(IGFBP2, IGF1R, cMET, EGFR, TGFa, EphA2, HER2, HER3, HER4, Table 4). In the presence of wild-type KRAS, high AREG mRNA levels were independently associated with $83.0 \%$ reduction in the risk of death compared to patients with AREG-low tumours. High EREG tumoural mRNA was a favourable outcome predictor for cetuximabtreated patients, irrespective of KRAS mutation status. High Ephrin A2 (EphA2) mRNA and advanced age were adverse independent prognosticators. Finally, among cetuximab- treated patients with AREG-low tumours, those with mutant KRAS fared significantly better than patients harbouring colon cancer with KRAS wild type $(81 \%$ decreased risk of death).

Impact of distinct types of KRAS and PIK3CA mutations on outcome of cetuximab-treated patients

We examined different types of KRAS and PIK3CA mutations for potentially distinct impact on patient 
Table 3 Prognostic/predictive factors at univariate analysis

\begin{tabular}{|c|c|c|c|c|c|}
\hline Predictive for survival & $\mathbf{N}$ & $\mathrm{HR}$ & $95 \% \mathrm{Cl}$ & $\begin{array}{l}\text { Median survival } \\
\text { (months) }\end{array}$ & $\begin{array}{l}\text { 2-sided } \\
\text { P value }\end{array}$ \\
\hline AREG mRNA (25th percentile) & & 0.47 & $0.31-0.70$ & & 0.0002 \\
\hline High & 124 & & & 29 & \\
\hline Low & 40 & & & 16 & \\
\hline EREG mRNA (75th percentile) & & 0.45 & $0.28-0.72$ & & 0.0009 \\
\hline High & 39 & & & 36 & \\
\hline Low & 117 & & & 23 & \\
\hline BRAF & & 8.1 & $3.44-19.0$ & & 0.00005 \\
\hline Mutation & 6 & & & 12 & \\
\hline Wild type & 208 & & & 28 & \\
\hline KRAS codon 12 & & 1.62 & $1.10-2.38$ & & 0.014 \\
\hline Mutation & 44 & & & 19 & \\
\hline Wild type & 129 & & & 29 & \\
\hline Predictive for Response & & $\begin{array}{l}\text { Odds Ratio } \\
\text { for Response }\end{array}$ & $95 \% \mathrm{Cl}$ & & 2-sided P \\
\hline AREG mRNA at 25th percentile & & 1.59 & $0.67-3.77$ & & 0.29 \\
\hline \multicolumn{6}{|l|}{ High vs. Low } \\
\hline EREG mRNA at 75th percentile & & 2.26 & $1.04-4.91$ & & 0.04 \\
\hline High vs. Low & & & & & \\
\hline
\end{tabular}

survival. Regarding KRAS, cetuximab-treated patients with codon 12 mutations had a median survival of 19 months (95\% CI 15-26), significantly lower $(\mathrm{p}=0.033)$ than the median survival of patients with other KRAS mutations (30 months, 95\% CI 20-35) and that of patients with wild-type KRAS (29 months, 95\% CI 2535). Further subgroup analyses showed that the median survival in patients with codon 12 KRAS mutations was also lower than the median survival of all other patient subgroups (other KRAS mutations or KRAS wild-type). Specifically, patients with codon 13 mutations reached a median survival of 28 months (95\% CI 16-39), those with other rarer KRAS mutations a median survival of 33 months (95\% CI 16-42) and patients with KRAS wild-type tumours a median survival of 29 months (95\% CI 25-35). Despite the rather small size of compared subgroups, a trend for statistical significance was evident $(\mathrm{p}=0.068$, Figure 4). No difference in patient outcomes was found when we compared tumours with PIK3CA exon 9 versus exon 20 mutations versus PIK3CA wildtype status, as survival times clustered from 25 to 29 months $(p=0.31)$. The only complex genotype that harboured significance for cetuximab benefit was CG3 $(\mathrm{p}=0.019)$ : Patients with tumours wild-type for all PIK3CA, KRAS, BRAF reached a median survival of 32 months (95\% CI 25-36), those with a PIK3CA mutation along with KRAS or BRAF mutation had a median survival of 26 months (95\% CI 16-32) while patients with any single mutation in KRAS, BRAF or PIK3CA genes had a median survival of 22 months (95\% CI 19-28). Apparently, concurrent mutations in PIK3CA along with KRAS or BRAF mutations carried rather weak additional adverse prediction.

\section{Discussion}

Despite exclusion of $30-40 \%$ of patients with KRASmutant tumours, cetuximab-based regimens fail in more than half of patients bearing KRAS wild-type colorectal cancer [5-10]. We sought to screen for additional predictive biomarkers in a retrospective clinical series of 226 cetuximab-treated advanced CRC patients and to look for hypothesis-generating hints of efficacy or resistance. Despite homogeneous management of all patients in standardised HeCOG recommended therapies, the retrospective nature of the translational research is an inherent limitation. Moreover, in the absence of a control arm with no treatment it is impossible to speculate on the prognostic (impact on outcome irrespective of therapy) or predictive (impact on benefit from cetuximab therapy) effect of the biomarkers studied. Finally, the administration of cetuximab across several lines of therapy combined with various chemotherapeutic agents constitutes an additional layer of heterogeneity, masking possible interactions and confounders. Moreover, survival analysis will not be robust and even tumor response may be influenced by the chemotherapy backbone to which cetuximab was added. Finally, it would be ideal for all of the tissue samples to have been collected prior to 


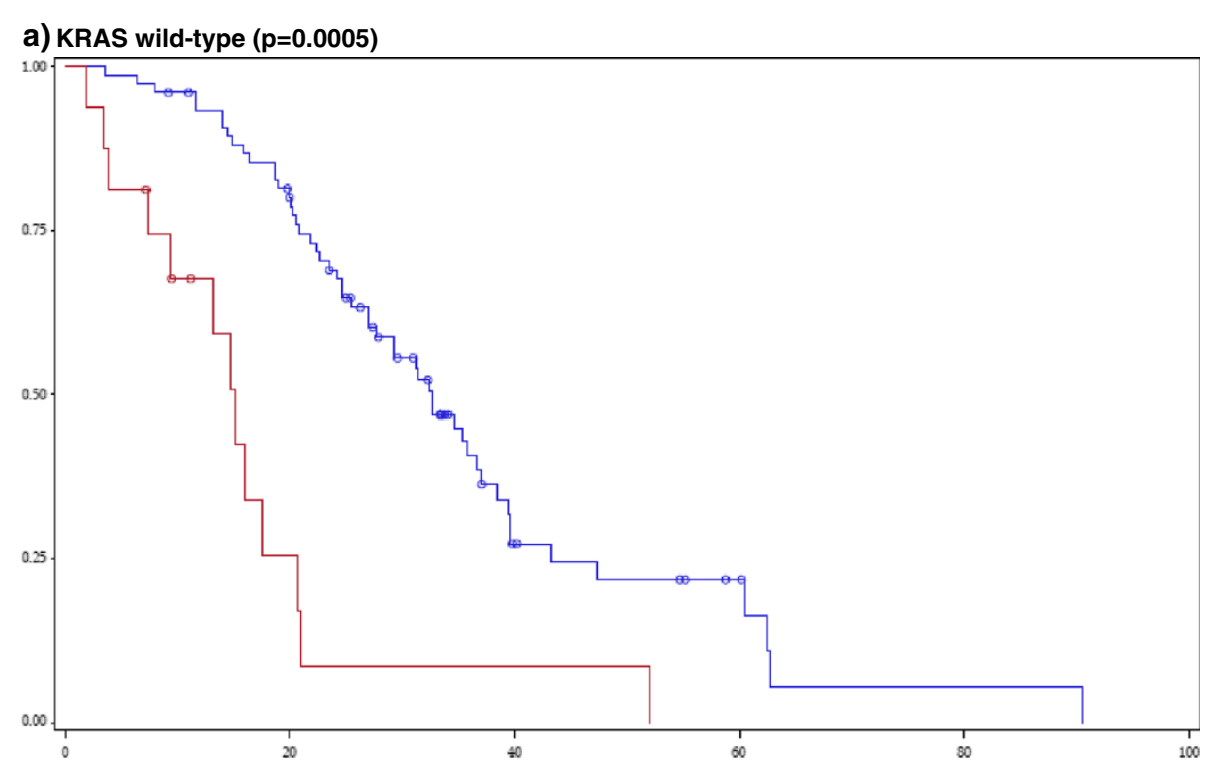

b) KRAS mutated $(p=0.64)$

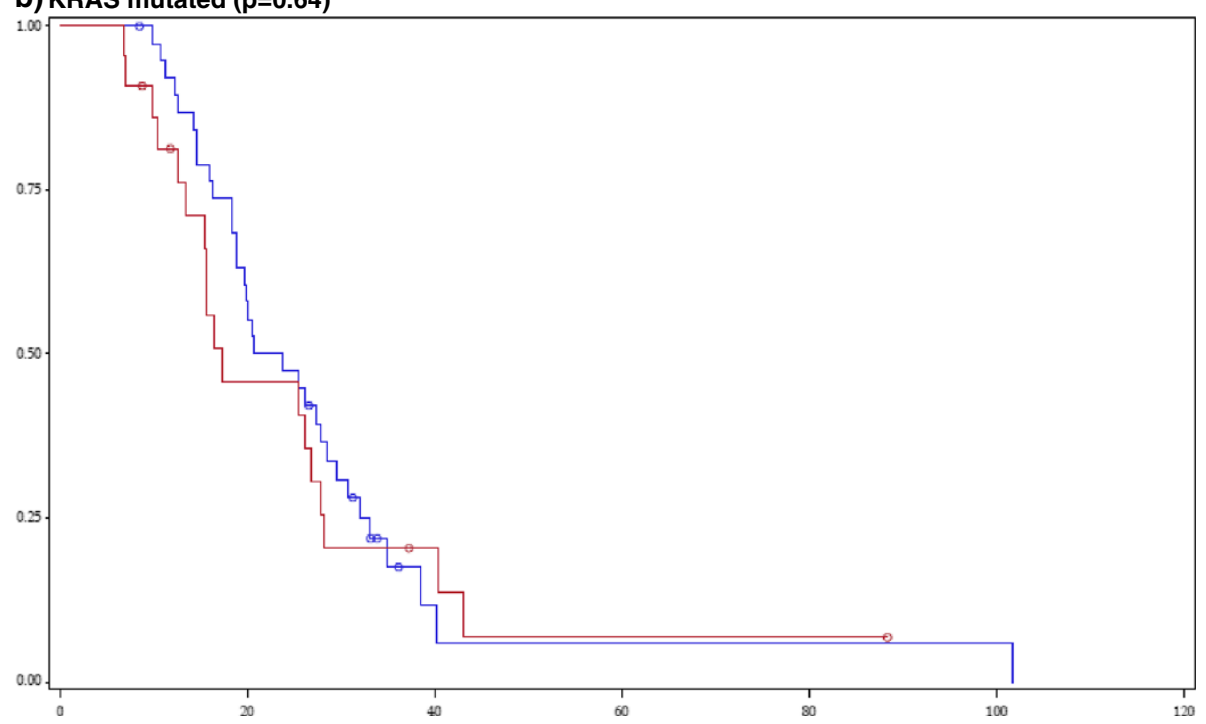

Figure 2 Impact of AREG mRNA levels on cetuximab-treated patient survival by KRAS tumour mutation. (Blue line: AREG-high, Red line: AREG-low). a) KRAS wild-type ( $p=0.0005)$, b) KRAS mutated ( $p=0.64)$.

cetuximab therapy, as some of the biomolecules studied may have changed by the time patients received 1st, 2nd or 3rd line therapy.

The incidence of observed KRAS, BRAF, NRAS, PIK3CA mutations are in line with those in the literature, ranging from $30-43 \%$ for KRAS, $0.5-7.2 \%$ for BRAF, $2-4 \%$ for NRAS, $5-17 \%$ for PIK3CA [8,16-19]. In univariate analysis, BRAF mutations were associated with an 8-fold increased risk of death, while KRAS mutations incurred a weaker, less than 2-fold increase in the risk of death only when codon 12 mutations were analysed separately. Similar to our findings, Souglakos et al. reported a markedly increased risk of death with a multivariate HR of 5.1 for BRAF mutations in cetuximab-treated CRC patients [20].
Moreover, Modest et al. recently reported median survival times of 23.5 months in KRAS wild-type, 18.9 months in KRAS codon 12-mutated and only 13 months in BRAFmutated CRC patients on cetuximab-based first-line therapy [21]. Seemingly, BRAF mutation seems to carry especially adverse prognosis in patients who receive anti-EGFR therapeutic modulation.

We observed no prognostic effect of NRAS, PIK3CA or KRAS mutations outwith codon 12. Despite initial reports of significant negative correlation between PIK3CA mutations and response to anti-EGFR moAbs, series by Prenen et al. and Saridaki et al. failed to find such an association $[10,18]$. De Roock et al. suggested that only exon 20 PIK3CA mutations were associated 


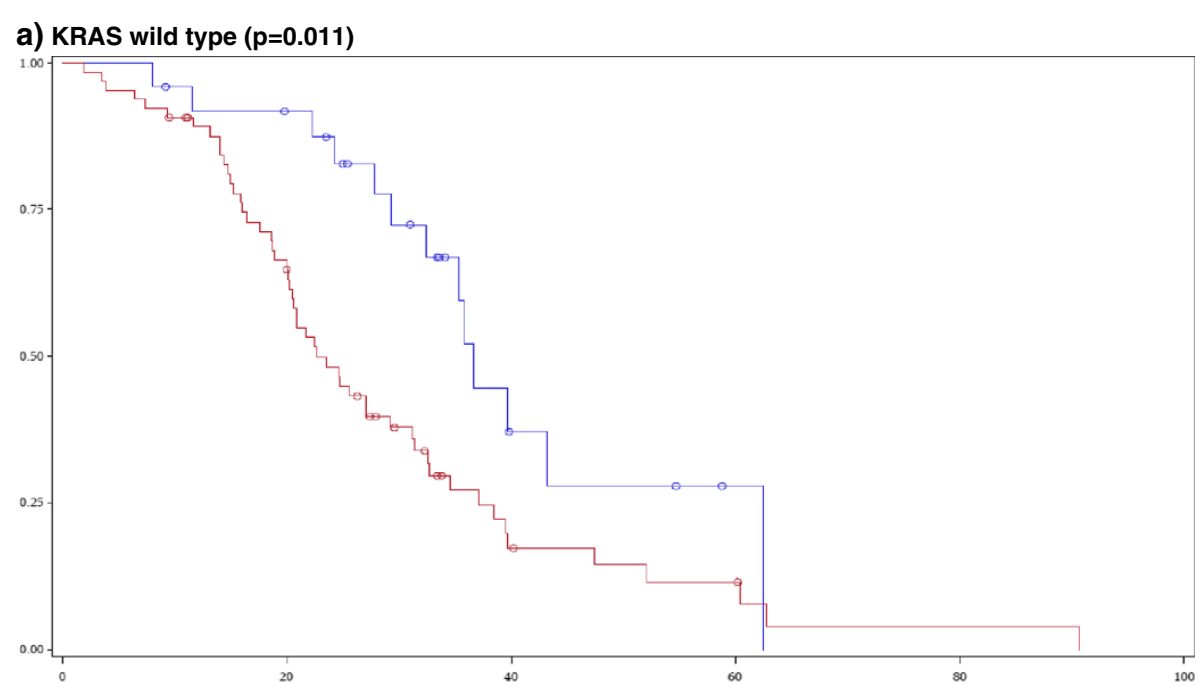

b) KRAS mutated $(\mathrm{p}=0.022)$

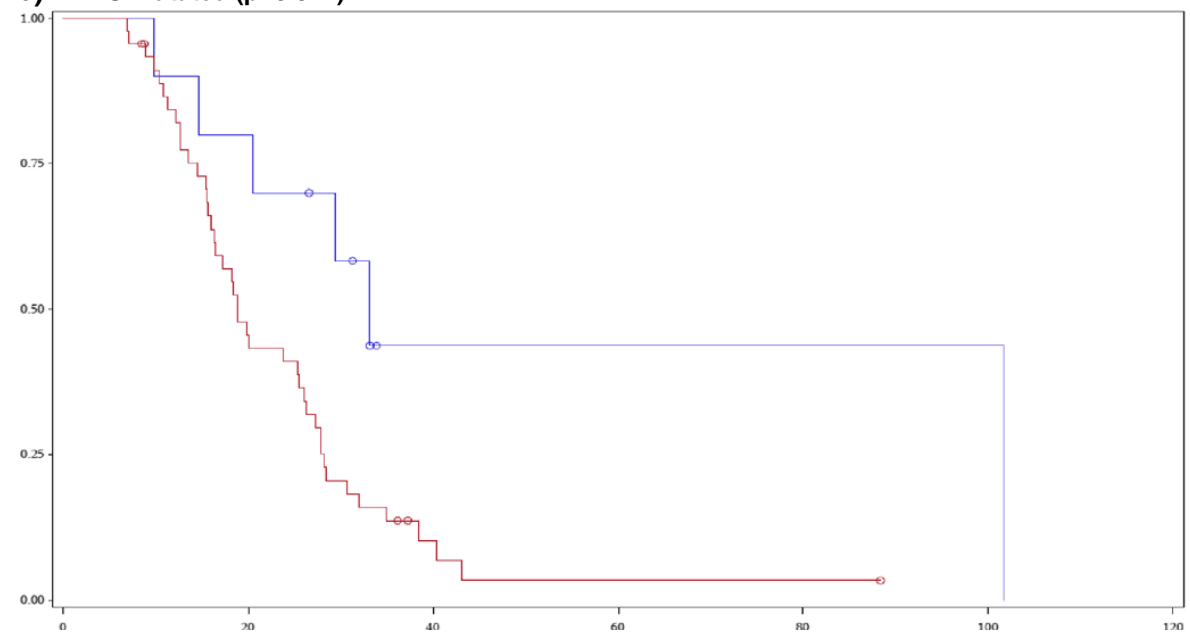

Figure 3 Impact of EREG mRNA levels on cetuximab-treated patient survival by KRAS tumour mutation. (Blue line: EREG-high, Red line: EREG-low). a) KRAS wild type ( $p=0.011)$, b) KRAS mutated ( $p=0.022$ ).

with resistance to cetuximab therapies [8,22]. In our series, only 5 tumours with exon 20 PIK3CA mutations were detected. Regarding KRAS, the same group reported in a pooled dataset of 579 cetuximab-treated CRC patients that those with codon 13 KRAS mutant tumours (G13D) had better outcomes (HR for death 0.50) compared to patients with other KRAS mutant tumours [9]. Those findings stood in our Greek population cohort, as the median survival of our cetuximab treated patients seemed to de-escalate from 29-33 months for KRAS wild type to 28 months for the KRAS codon 13 mutated group, dropping to 19 months for the KRAS codon 12 mutated group. Although Peeters et al. recently failed to find a prognostic difference between codon 12 and codon 13 KRAS mutations in CRC patients managed with panitumumab in three randomised trials, another group from Germany did so for cetuximab [21,23,24]. KRAS codon 12 mutant
CRC patients reached a median survival of only 18.9 months versus 23.5 months for KRAS wild type and 26.2 months for KRAS codon 13 mutant CRC patients on first line cetuximab+CAPIRI/CAPOX. Indeed in preclinical experiments, transforming potential, RAS/GTP activation, MAPK phosphorylation and tumour growth were markedly increased in the presence of KRAS codon 12 mutations, followed in decreasing order by codon 13, 61, 117 and 146 mutations $[25,26]$. Moreover, microarray profiling of multigene expression signatures associated with KRAS mutations revealed that codon 12 mutations were linked to a gene cluster distinct from all other KRAS mutations [25]. Further validation of these data is warranted in order to confirm or refute the hypothesis that specific molecular characteristics of KRAS mutations are determinants of cetuximab benefit.

We observed a strong correlation of increased tumoural mRNA of AREG, EREG with clinical benefit 
Table 4 Prognostic/predictive factors at multivariate analysis

\begin{tabular}{lccc}
\hline Survival (N=136) & HR & $\mathbf{9 5 \% ~ C l}$ & Wald-p \\
\hline Age & 1.64 & $1.08-2.48$ & 0.0199 \\
$>$ median & & & \\
EPHA2 & 1.67 & $1.05-2.63$ & 0.0287 \\
High vs. Low & & & \\
EREG & 0.38 & $0.21-0.66$ & 0.0006 \\
High vs. Low & 7.98 & $2.98-21.40$ & $<.0001$ \\
AREG/KRAS interaction & & & \\
AREG high in KRAS mutant vs & & & \\
AREG high in KRAS wild type & & & \\
AREG & & & \\
AREG high vs. low for KRAS wild type & 0.17 & $0.08-0.34$ & $<.0001$ \\
AREG high vs. low for KRAS mutant & 1.33 & $0.67-2.61$ & 0.4165 \\
KRAS & & & \\
KRAS mutant vs. wild type for AREG high & 1.49 & $0.90-2.45$ & 0.1184 \\
KRAS mutant vs. wild type for AREG low & 0.19 & $0.08-0.43$ & $<.0001$ \\
\hline
\end{tabular}

from cetuximab ( $>50 \%$ reduction in the risk of death), in keeping with data reported by four other groups $[11,20,27,28]$. Gene expession of the two ligands, which are collocalised on chromosome 4q13.3 and produced by tumour cells in autocrine fashion, was tightly correlated and occurred more often in KRAS and BRAF wild type tumours. The correlation of low EREG/AREG expression with KRAS or BRAF mutated status could be due to the constitutive activation of the RAS/RAF/ MAPK pathway which makes activation of the EGFR pathway redundant biologically. Alternatively, it could be due to a negative feedback loop linking MAPK axis activation with suppression of the EGFR pathway.

Objective response to anti-EGFR therapy was correlated to continuous expression levels of AREG, EREG but only to categorical EREG mRNA distribution, intriguingly more strongly in KRAS mutated cases. The smooth nature of relationship between marker levels and outcome, resulting in lack of a universal cut-point applicable in all clinical situations has already been reported by Jacobs et al. [11]. In the presence of downstream activation of the EGFR/RAS/MAPK axis due to mutated KRAS effector, gene expression of AREG, EREG ligands would be biologically irrelevant for benefit from cetuximab. In our series this was the case for AREG, however tumour EREG mRNA retained predictive significance for survival both in KRAS wild-type as well as mutated cases, a counterintuitive finding, further supported by the predictive significance of EREG for response, even in KRAS mutant patients. AREG and EREG are not biologically identical: AREG binds EGFR only, whereas EREG binds EGFR and HER4 and leads to a prolonged state of receptor activation [29]. Compared to AREG, tumour EREG mRNA expession was a stronger predictor of cetuximab benefit in KRAS wild type cases in three more series $[13,20,27]$. Consequently, we may speculate that even in the presence of KRAS mutations, cetuximab binding to EGFR prevents high levels of EREG from activating HER1/HER4 heterodimers and thus abrogates signalling pathways distinct from RAS/MAPK. In fact, Baker et al. reported HER4 gene expression as one of the genes significantly associated with clinical benefit in 144 cetuximab-treated CRC patients [28]. However, our findings should be interpreted with caution as the

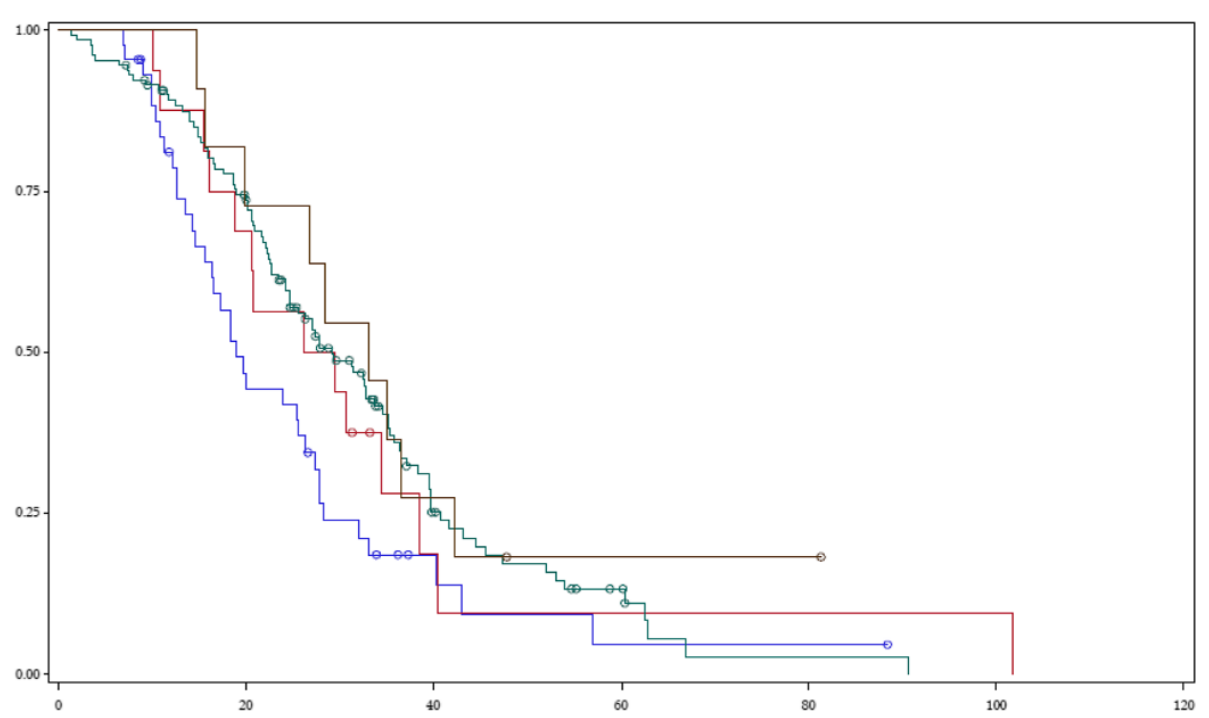

Figure 4 Survival of cetuximab-treated patients by type of KRAS mutation in tumour. Blue line: codon 12 mutations, Red line: codon 13 mutations, Green line: wild-type, Purple line: other KRAS mutations. 
number of EREG-high, KRAS-mutant cases analysed was low $(\mathrm{n}=10)$ and random effects cannot be excluded.

In our cohort, the survival of cetuximab-treated patients with KRAS wild type, AREG-low CRC (median 15 months) was as poor as the survival of patients with KRAS mutant tumours (17-22 months). If this finding is confirmed in independent series, AREG expression in KRAS wild type cases would emerge as a robust biomarker of cetuximab efficacy. Baker et al. suggested the use of a 4-gene score (AREG, EREG, DUSP6, SLC26A3) for the prediction of anti-EGFR treatment benefit in KRAS wild type CRC [28]. In the presence of low AREG and thus inactive EGFR pathway, cetuximab binding to HER dimers might elicit a paradoxical "pro-survival» cellular response via MAPK-dependent and MAPK-independent pathways, as suggested by Oliveras-Ferraros et al. [30]. Finally, the markedly increased risk of death (8-fold) of high AREG mRNA in KRAS mutant versus KRAS wild-type CRC patients in multivariate analysis could be explained if AREG is viewed as a protein regulated by KRASdependent transcription factors: the former group includes tumours bearing KRAS mutations with a markedly pro-survival, proliferative activating effect, whereas the latter group includes KRAS wild type tumours addicted to active EGFR signalling that is amenable to abrogation by cetuximab.

\section{Conclusions}

Our study confirms published data on the prognostic/predictive significance of BRAF, KRAS mutations and on the tumour expression of AREG, EREG mRNA in cetuximabtreated patients with colorectal cancer. Moreover, we present data suggesting a) a differential impact of noncodon 12 KRAS mutations on outcome of cetuximabtreated patients of Greek ethnic origin, b) a KRAS mutation-independent predictive significance of EREG mRNA expression, c) a strong predictive value of AREG mRNA expression in KRAS wild type patients. These findings warrant independent validation.

\section{Competing interests}

The authors declare that they have no competing interests.

\section{Authors' contributions}

GP: Conceived of the study and drafted the manuscript. VK: Carried out the molecular genetic studies. WDR, ST: Carried out the mutational testing. GK: Performed the statistical analysis. DT: Reviewed histologically the tumour samples. PP, TM, DP, IX, JS, GK, ACT, ER, EG, DB, IE, IB, DP, NP, GF: Participated in the design and coordination of the study. All authors read and approved the final manuscript.

\section{Author details}

'Department of Medical Oncology, loannina University Hospital, loannina, Greece. ${ }^{2}$ Department of Pathology, Aristotle University of Thessaloniki School of Medicine, Thessaloniki, Greece. ${ }^{3}$ Laboratory of Molecular Oncology, Hellenic Foundation for Cancer Research, Aristotle University of Thessaloniki School of Medicine, Thessaloniki, Greece. ${ }^{4}$ Department of Oncology, Ziekenhuis Oost-Limburg, Genk, Belgium. ${ }^{5}$ Health Data Specialists Ltd, Athens, Greece. 'Department of Medical Oncology, "Hippokration" Hospital,
Athens, Greece. ${ }^{7}$ Division of Oncology, Department of Medicine, University Hospital, University of Patras Medical School, Patras, Greece. ${ }^{8}$ Bank of Cyprus Oncology Center, Nicosia, Cyprus. 'Department of Medical Oncology, "Papageorgiou" Hospital, Aristotle University of Thessaloniki School of Medicine, Thessaloniki, Greece. ${ }^{10}$ Third Department of Medical Oncology, "Agii Anargiri" Cancer Hospital, Athens, Greece. ${ }^{11}$ Department of Pathology, Ippokration Hospital, Athens, Greece. ${ }^{12}$ Department of Pathology, University Hospital, University of Patras Medical School, Patras, Greece. ${ }^{13}$ Third Department of Medical Oncology, "Hygeia" Hospital, Athens, Greece. ${ }^{14}$ Second Department of Medical Oncology, "Metropolitan" Hospital, Piraeus, Greece. ${ }^{15}$ First Department of Medical Oncology, "Metropolitan" Hospital, Piraeus, Greece. ${ }^{16}$ Department of Pathology, "Papageorgiou" Hospital, Thessaloniki, Greece. ${ }^{17}$ Oncology Department, General Hospital of Chania, Crete, Greece. ${ }^{18}$ Oncology Section, Second Department of Internal Medicine, "Hippokration" Hospital, Athens, Greece.

Received: 1 September 2012 Accepted: 30 January 2013 Published: 2 February 2013

\section{References}

1. Jemal A, Siegel R, Ward E, et al: Cancer Statistics, 2009. CA Cancer J Clin 2009, 59:225-249.

2. Siena S, Sartore-Bianchi A, Di Nicolantonio F, et al: Biomarkers Predicting Clinical Outcome of Epidermal Growth Factor Receptor-Targeted Therapy in Metastatic Colorectal Cancer. J Natl Cancer Inst 2009, 101:1308-1324.

3. Linardou H, Briasoulis E, Dahabreh IJ, et al: All about KRAS for clinical oncology practice: gene profile, clinical implications and laboratory recommendations for somatic mutational testing in colorectal cancer. Cancer Treat Rev 2011, 37:221-233.

4. Scheffzek K, Ahmadian MR, Kabsch W, et al: The Ras-RasGAP complex: structural basis for GTPase activation and its loss in oncogenic Ras mutants. Science 1997, 277:333-338.

5. Amado RG, Wolf $M$, Peeters $M$, et al: Wild-type KRAS is required for panitumumab efficacy in patients with metastatic colorectal cancer. $J$ Clin Oncol 2008, 26:1626-1634.

6. Van Cutsem E, Kohne $\mathrm{CH}$, Hitre E, et al: Cetuximab and chemotherapy as initial treatment for metastatic colorectal cancer. N Engl J Med 2009, 360:1408-1417.

7. Van Cutsem E, Kohne CH, Lang l, et al: Cetuximab plus irinotecan, fluorouracil, and leucovorin as first-line treatment for metastatic colorectal cancer: updated analysis of overall survival according to tumor KRAS and BRAF mutation status. J Clin Oncol 2011, 29:2011-2019.

8. De Roock W, Claes B, Bernasconi D, et al: Effects of KRAS, BRAF, NRAS, and PIK3CA mutations on the efficacy of cetuximab plus chemotherapy in chemotherapy-refractory metastatic colorectal cancer: a retrospective consortium analysis. Lancet Oncol 2010, 11:753-762.

9. De Roock W, Jonker DJ, Di Nicolantonio F, et al: Association of KRAS p.G13D mutation with outcome in patients with chemotherapy-refractory metastatic colorectal cancer treated with cetuximab. JAMA 2010, 304:1812-1820.

10. De Roock W, Piessevaux H, De Schutter J, et al: KRAS wild-type state predicts survival and is associated to early radiological response in metastatic colorectal cancer treated with cetuximab. Ann Oncol 2008, 19:508-515.

11. Jacobs B, De Roock W, Piessevaux H, et al: Amphiregulin and epiregulin mRNA expression in primary tumors predicts outcome in metastatic colorectal cancer treated with cetuximab. J Clin Oncol 2009, 27:5068-5074.

12. Sanchez-Navarro I, Gamez-Pozo A, Gonzalez-Baron M, et al: Comparison of gene expression profiling by reverse transcription quantitative PCR between fresh frozen and formalin-fixed, paraffin-embedded breast cancer tissues. Biotechniques 2010, 48:389-397.

13. Koutras AK, Kalogeras KT, Dimopoulos MA, et al: Evaluation of the prognostic and predictive value of HER family mRNA expression in highrisk early breast cancer: a Hellenic Cooperative Oncology Group (HeCOG) study. Br J Cancer 2008, 99:1775-1785.

14. Nishino M, Jagannathan JP, Ramaiya NH, Van den Abbeele AD, Revised RECIST: guideline version 1.1: What oncologists want to know and what radiologists need to know. AJR Am J Roentgenol 2010, 195:281-289.

15. Miller R, Siegmund D: Maximally Selected Chi-Square Statistics. Biometrics 1982, 38:1011-1016

16. Sartore-Bianchi A, Martini M, Molinari F, et al: PIK3CA Mutations in Colorectal Cancer Are Associated with Clinical Resistance to EGFRTargeted Monoclonal Antibodies. Cancer Res 2009, 69:1851-1857. 
17. Siena S, Sartore-Bianchi A, Di Nicolantonio F, Balfour J, Bardelli A: Biomarkers predicting clinical outcome of epidermal growth factor receptor-targeted therapy in metastatic colorectal cancer. J Natl Cancer Inst 2009, 101(19):1308-1324.

18. Prenen $H$, De Schutter J, Jacobs B, et al: PIK3CA mutations are not a major determinant of resistance to the epidermal growth factor receptor inhibitor cetuximab in metastatic colorectal cancer. Clin Cancer Res 2009, 15:3184-3188.

19. Perrone F, Lampis A, Orsenigo M, et al: PI3KCA/PTEN deregulation contributes to impaired responses to cetuximab in metastatic colorectal cancer patients. Ann Oncol 2009, 20:84-90.

20. Saridaki Z, Tzardi M, Papadaki C, et al: Impact of KRAS, BRAF, PIK3CA mutations, PTEN, AREG, EREG expression and skin rash in $>/=2$ line cetuximab-based therapy of colorectal cancer patients. PLoS One 2011, 6:e15980.

21. Modest DP, Jung A, Moosmann N, et al: The influence of KRAS and BRAF mutations on the efficacy of cetuximab-based first-line therapy of metastatic colorectal cancer: An analysis of the AIO KRK-0104-trial. Int J Cancer 2012, 131(4):980-986.

22. De Roock W, De Vriendt V, Normanno N, et al: KRAS, BRAF, PIK3CA, and PTEN mutations: implications for targeted therapies in metastatic colorectal cancer. Lancet Oncol 2011, 12:594-603.

23. Douillard JY, Siena S, Cassidy J, et al: Randomized, phase III trial of panitumumab with infusional fluorouracil, leucovorin, and oxaliplatin (FOLFOX4) versus FOLFOX4 alone as first-line treatment in patients with previously untreated metastatic colorectal cancer: the PRIME study. J Clin Oncol 2010, 28:4697-4705.

24. Peeters M: Evaluation of Individual Codon 12 and 13 Mutant (MT) KRAS Alleles as Prognostic and Predictive Biomarkers of Response to Panitumumab (pmab) in Patients with Metastatic Colorectal Cancer. ECCO 2011, 2011:20.

25. Smith $G$, Bounds $R$, Wolf $H$, et al: Activating K-Ras mutations outwith 'hotspot' codons in sporadic colorectal tumours - implications for personalised cancer medicine. Br J Cancer 2010, 102:693-703.

26. Janakiraman M, Vakiani E, Zeng Z, et al: Genomic and biological characterization of exon 4 KRAS mutations in human cancer. Cancer Res 2010, 70:5901-5911.

27. Khambata-Ford S, Garrett CR, Meropol NJ, et al: Expression of epiregulin and amphiregulin and K-ras mutation status predict disease control in metastatic colorectal cancer patients treated with cetuximab. J Clin Oncol 2007, 25:3230-3237.

28. Baker JB, Dutta D, Watson D, et al: Tumour gene expression predicts response to cetuximab in patients with KRAS wild-type metastatic colorectal cancer. Br J Cancer 2011, 104:488-495.

29. Shelly M, Pinkas-Kramarski R, Guarino BC, et al: Epiregulin is a potent panErbB ligand that preferentially activates heterodimeric receptor complexes. J Biol Chem 1998, 273:10496-10505.

30. Oliveras-Ferraros C, Vall-Llovera AM, Salip DC, et al: Evolution of the predictive markers amphiregulin and epiregulin mRNAs during longterm cetuximab treatment of KRAS wild-type tumor cells. Invest New Drugs 2012, 30:846-852.

\section{doi:10.1186/1471-2407-13-49}

Cite this article as: Pentheroudakis et al:: Biomarkers of benefit from cetuximab-based therapy in metastatic colorectal cancer: interaction of EGFR ligand expression with RAS/RAF, PIK3CA genotypes. BMC Cancer 2013 13:49.

\section{Submit your next manuscript to BioMed Central and take full advantage of:}

- Convenient online submission

- Thorough peer review

- No space constraints or color figure charges

- Immediate publication on acceptance

- Inclusion in PubMed, CAS, Scopus and Google Scholar

- Research which is freely available for redistribution 